"C 2019 IEEE. Personal use of this material is permitted. Permission from IEEE must be obtained for all other uses, in any current or future media, including reprinting/republishing this material for advertising or promotional purposes, creating new collective works, for resale or redistribution to servers or lists, or reuse of any copyrighted component of this work in other works." 


\section{Some Convolution and Scale Transformation Techniques to Enhance GPR Images}

\author{
Nalika Ulapane \\ Electrical and Electronic Engineering \\ The University of Melbourne \\ Parkville VIC 3010, Australia \\ nalika.ulapane@unimelb.edu.au
}

\author{
Lasitha Piyathilaka \\ Centre for Autonomous Systems \\ University of Technology Sydney \\ Ultimo NSW 2007, Australia \\ lasitha.piyathilaka@uts.edu.au
}

\author{
Sarath Kodagoda \\ Centre for Autonomous Systems \\ University of Technology Sydney \\ Ultimo NSW 2007, Australia \\ sarath.kodagoda@uts.edu.au
}

\begin{abstract}
Locating reinforcement rods embedded inside concrete wall-like structures, as well as locating subsurface features such as voids, cracks, and interfaces is an essential part of structural health monitoring of concrete infrastructure. The Ground Penetrating Radar (GPR) technique has been commonly used as a means of Non-destructive Testing and Evaluation (NDT \& E) which suits the purpose. In the recent past, the interest of using GPR to assess the crowns (i.e., top) of concrete sewers has been rising. Moisture is well known to be a challenge for GPR imaging as moisture tends to influence GPR waves. This challenge becomes more common and persistent inside sewers since sewer walls contain considerable surface and subsurface moisture as a result of the humid environment created by the waste water flowing through sewers as well as the bacteria and gas induced acid attacks. Forming a part of sewer condition assessment-related research with the objective of assessing moist concrete, this paper presents some preliminary results which demonstrate how some simple scale transformations and convolution can help in enhancing GPR images in grey-scale. A set of raw GPR signals captured on a moist concrete block inside a laboratory environment is considered. The effect of enhancement is demonstrated against a benchmark image constructed by mapping the raw signals directly onto grey-scale.

Index Terms - concrete, GPR, NDT, NDE, radar, sensor, sewer
\end{abstract}

\section{INTRODUCTION}

Ground Penetrating Radar (GPR) is a rapidly growing geophysical sensing method [1] that has seen tremendous progress in the development of theory, technique, technology, and range of applications over the past three decades [2]. Applications of this method range from geology-related and geotechnical studies [2], ground water-related studies [2, 3, 4], sedimentalogy [2, 5], glaciology [2], archaeology [2], and Non-Destructive Testing and Evaluation (NDT\&E) [2, 6].

In the domain of NDT\&E-which is the interest of this paper, concrete inspection becomes a common target application of GPR [6, 7, 8]. With the recent interest in research relating to concrete sewer condition assessment [9, 10, 11, 12, 13, 14, 15], interest towards the use of GPR to assess the crown (i.e., top) of concrete sewers has also increased [16, 17]. However, sewer walls are understandably quite moist due to the humid atmosphere prevalent inside sewers, which is caused by the waste water flow and the influence of bacteria and gas induced acid attacks. As a result of this moisture, it can be challenging to obtain good quality GPR data inside sewers, as moisture is known to influence the amplitude of GPR waves [6]. This added challenge with moisture for example, is an instance which demands for additional techniques in the signal processing front to enhance GPR images through some post-processing applied on raw GPR signals.

Conventionally, GPR imaging is done using Synthetic Aperture Radar (SAR) [18, 19] and Migration techniques [20, 21, 22]. Some further GPR signal enhancement techniques include the likes of seismic analysis techniques [23], non-linear data processing techniques [24], wave-equation redatuming [25], sliding-window space-frequency matrices [26], and deep learning-based techniques [17, 27].Moreover, some other research have focused on using GPR for detecting chloride and evaluating the moisture content in concrete [28, 29].However, work that closely relate to applications such as sewer condition assessment and focus on detecting objects (e.g., reinforcements rods) or physical anomalies such as voids, cracks, and interfaces through enhancing GPR waves adversely impacted by any moisture present in concrete are rare. Therefore, this paper makes a contribution by focusing on enhancing GPR images to better visualize subsurface objects and defects embedded inside moist concrete, with the target application of concrete sewer condition assessment in mind.

The paper is structured as follows: Section [II presents the fundamental principles related to the work of this paper; section III] describes the experimental set-up used; the GPR image enhancement techniques experimented along with results are presented in section [V] and section $\mathrm{V}$ concludes the paper while discussing implications of results and avenues for future work.

\section{RELEVANT PRINCIPLES}

GPR acquires data by emitting radar waves into a test piece and recording echo waves reflected by sub-surface objects, defects, or changes of certain material properties. References [1, 2] will be useful to gain in-depth understanding about GPR. The primary output produced by a GPR device is known as the A-Scan matrix, which is a collection of time domain reflected echo waves. Fig. 1 illustrates the standard scenario of operation of a GPR antenna. 


\section{A. GPR A-Scan Matrix}

When a sample distance $d x\left(\in \Re^{+}\right)$is specified and a GPR antenna is moved on the surface of a test piece along a straight line for a distance $X\left(\in \Re^{+}, X>d x\right)$ as shown in Fig. 1 at every spatial point denoted as $x_{i}\left(1 \leq i \leq n ; i, n \in \mathbb{Z}^{+}\right)$along the straight line such that $x_{i+1}-x_{i}=d x \forall i$, the antenna records a time domain echo wave $S_{i}(t)$. All $S_{i}(t)$ waves can be considered row vectors of the same length (assume vector size to be $1 \times m, m \in \mathbb{Z}^{+}$) as all waves will have equal signal duration and sampling frequency. An example for an $S_{i}(t)$ wave is shown in Fig. 2 The A-Scan matrix (denoted herein as $A$, a matrix of size $m \times n$ ) is constructed by arranging all $S_{i}(t)$ waves captured along a straight line in the following manner:

$$
A=\left[S_{1}(t)^{T}, S_{2}(t)^{T}, \ldots ., S_{i}(t)^{T}, \ldots, S_{n}(t)^{T}\right]
$$

\section{B. GPR B-Scan Image}

B-Scan images can be constructed by applying a variety of migration techniques on A-Scans, after A-Scan waves have gone through some signal processing and conditioning steps [20, 21, 22]. Fig. 3 shows an example for the construction of a B-Scan image, and Fig. 4 shows an example real-world B-Scan image captured as part of this work.

For the work of this paper, migrating of processed A-Scan matrices to B-Scan images is done simply by mapping the processed A-Scan matrices to grey-scale images. The values in a processed A-Scan matrix are quantized to 256 equal levels and the quantized level corresponding to each cell in

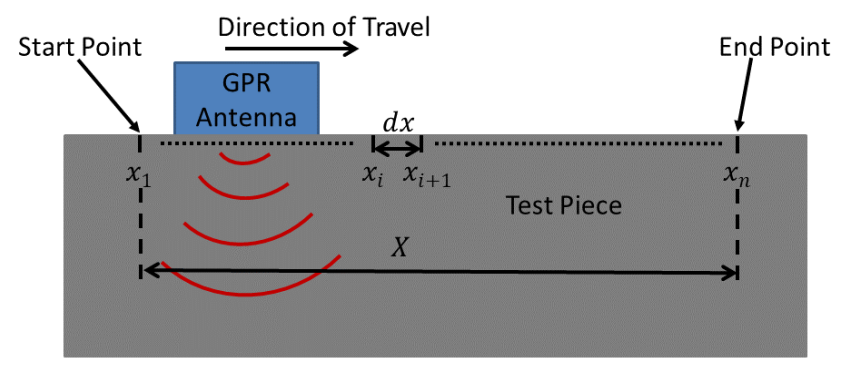

Fig. 1. Standard operation scenario of a GPR antenna.

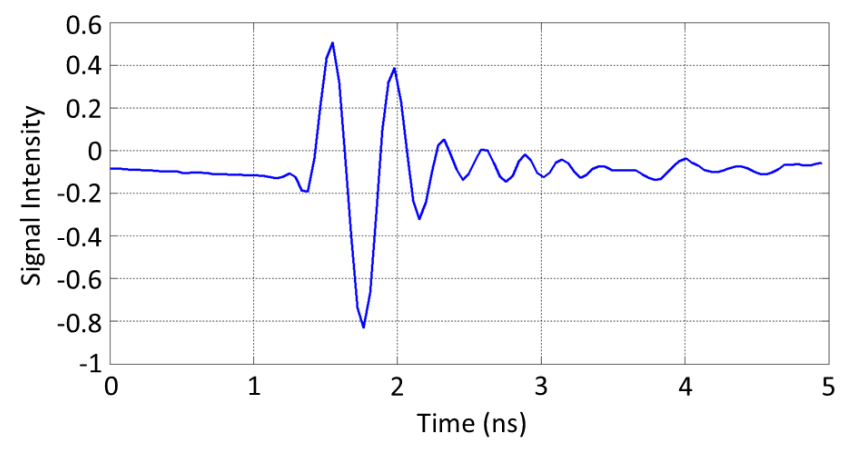

Fig. 2. An example real-world GPR echo wave found in an A-Scan.

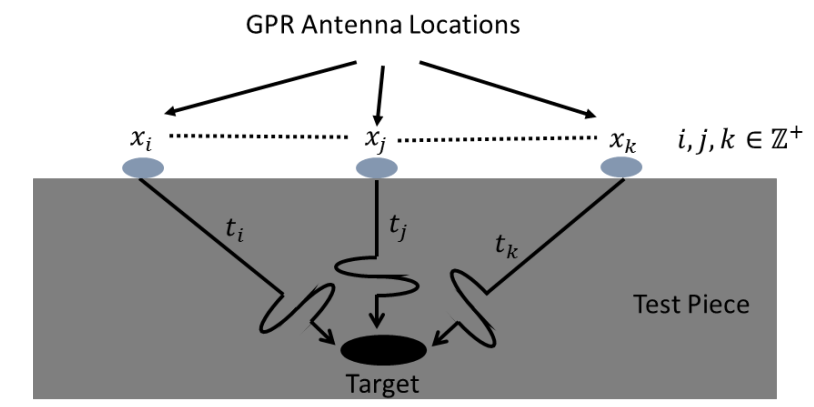

$t_{j}<t_{i} ; t_{j}<t_{k} ; t_{i}, t_{j}, t_{k} \in \mathbb{R}^{+}$

$t_{i}, t_{j}, t_{k}$ Are the times taken for the wave to reflect from the target when the antenna is located at points $x_{i}, x_{j}, x_{k}$
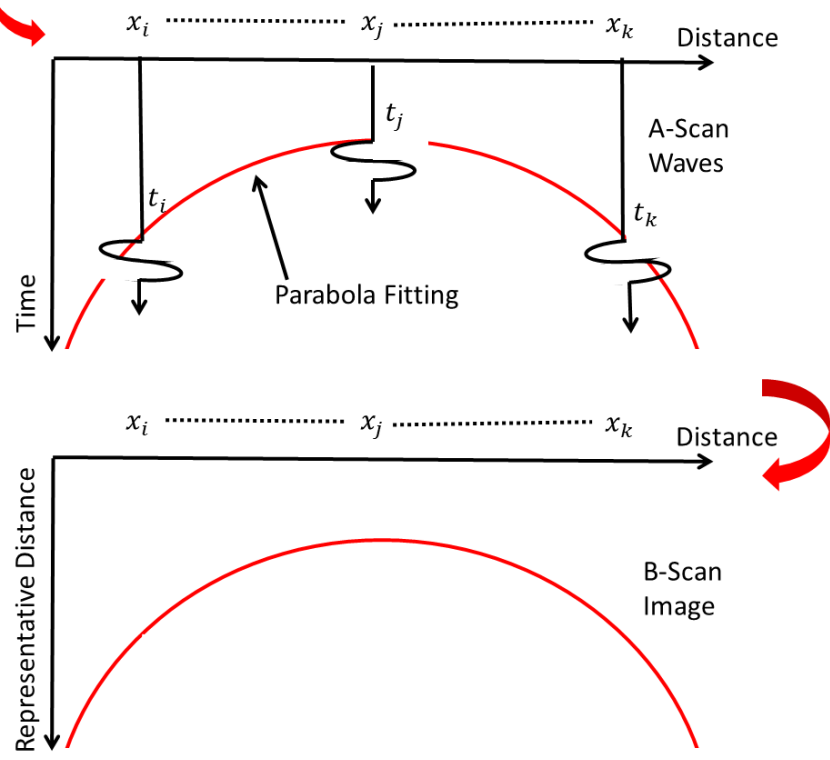

Fig. 3. Example B-Scan image construction.

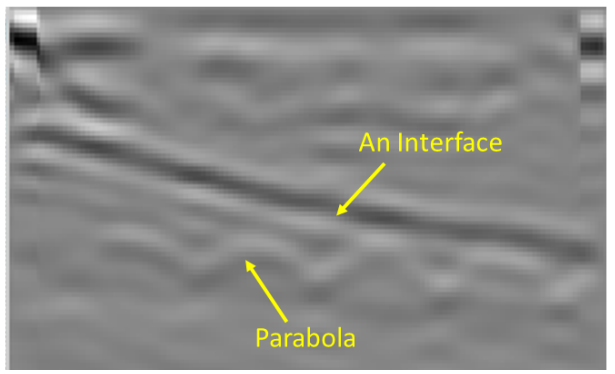

Fig. 4. An example for a real-world B-Scan image.

the processed A-Scan matrix is then mapped as a pixel in grey-scale generating the B-Scan image, where the highest quantization level corresponds to white and the lowest level corresponds to black.

\section{Probability Density Function (PDF) of Normal Distribu- tion}

For a continuous random variable $x \in \Re$ (note that the $x$ notation here is used for a random variable, and does 
not coincide with the $x, X$ notations used before to denote distance), the standard definition of the PDF of the normal distribution comes as:

$$
P(x)=\frac{1}{\sigma \sqrt{2 \pi}} e^{-(x-\mu)^{2} / 2 \sigma^{2}},
$$

where $\mu$ and $\sigma$ are the mean and standard deviation respectively, of the distribution.

\section{D. $2 D$ Convolution}

When a 2D matrix $g$ and a 2D kernel $h$ are available, the standard definition of the discrete 2D convolution output matrix $y$ with indices denoted by $a, b$ comes as:

$$
\begin{gathered}
y[a, b]=g[a, b] * h[a, b] \\
g[a, b] * h[a, b]=\sum_{j=-\infty}^{\infty} \sum_{i=-\infty}^{\infty} g[i, j] h[a-i, b-j]
\end{gathered}
$$

\section{EXPERIMENTAL SET-UP}

Fig. 5 shows the experimental set-up used for the work of this paper. GPR data were captured using the MALA CX12 ${ }^{\circ}$ Concrete Scanner $2.6 \mathrm{GHz}$ antenna (http:// www.malagpr.com.au/_-date of last visit: December 29, 2018) shown in Fig. 5(c). A cross section of the test piece along the GPR antenna's direction of travel is shown in Fig. 5(d). The test piece is mimicking a concrete sewer wall; it has steel reinforcement rods embedded within perpendicular to the direction of travel of the antenna, and has a top layer made of Gypsum-mimicking a layer of corroded concrete on the outer surface. The Gypsum layer was made to have a slope with the expectation of it being more obvious to identify form GPR B-Scans. To mimic moisture inside sewer walls, the test piece was left immersed in water for 48 hours as shown in Fig. 5(a), and was allowed to dry at room temperature for 24 hours as shown in Fig. 5.b) before taking GPR measurements. After 24 hours, when the test piece was moist to an extent closely comparable with a sewer crown, GPR measurements were recorded by moving the antenna as shown in Fig. 5(c).

\section{Methodology and Results}

\section{A. Pre-processing}

When the GPR A-Scan matrix captured after the test piece dried for 24 hours was directly mapped to a grey-scale image as explained in subsection II-B the resulting B-Scan image happened to be the one in Fig. 6(a). At the top of Fig. 6a), some horizontal lines are visible. These lines are a result of the initial reflection (or the background reflection) caused by the interface where the antenna touches the top of the Gypsum layer, and this reflection is not necessary for analysis and is undesired as what we are interested in are subsurface features. Therefore, it is good practice to remove this background reflection. Subtracting the background reflection was done by performing the following operation on the A-Scan matrix:

$$
A_{b r}=A-\left[\frac{1}{n} \sum_{i=1}^{n} S_{i}(t)^{T}\right][1, \ldots, 1, \ldots, 1]_{1 \times n},
$$

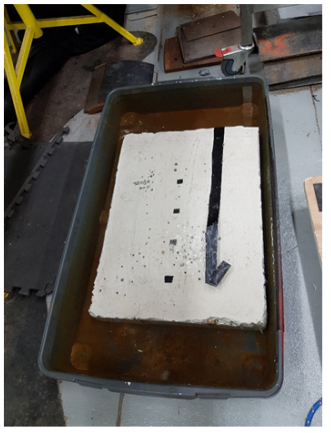

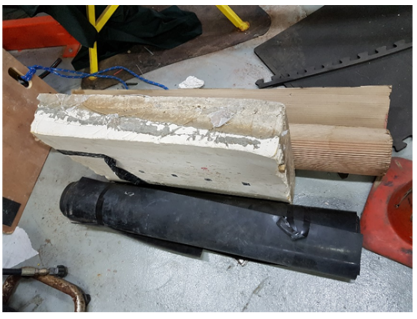

(b) (a)

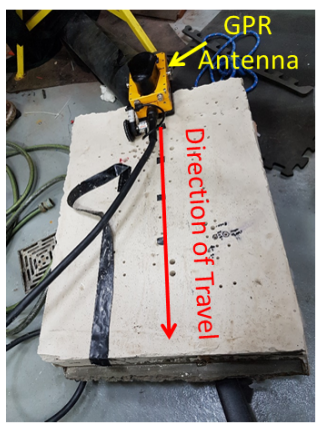

(c)

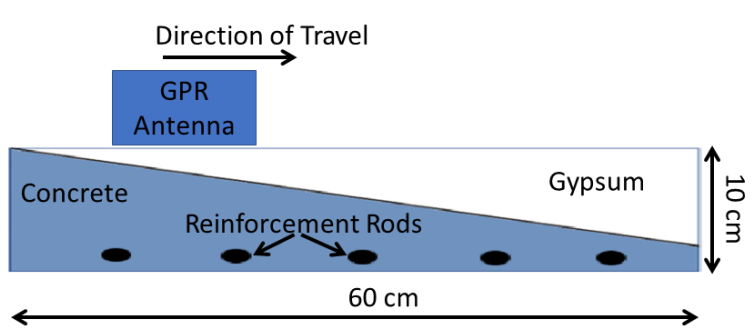

(d)

Fig. 5. Experimental set-up: (a) Test piece immersed in water for 48 hours; (b) Test piece drying at room temperature for 24 hours; (c) Antenna movement for capturing GPR measurements; (d) Cross section of the test piece along the direction of travel of the GPR antenna.

where $A_{b r}$ is the background removed A-Scan matrix and $A, i, n$ and $S_{i}(t)$ are consistent with eq. 1 . When $A_{b r}$ was converted to its B-Scan, what resulted was the image in Fig. 6(b) which does not have the horizontal lines at the top unlike in Fig. 6(a). This B-Scan image in Fig. 6(b) obtained from $A_{b r}$ is considered the benchmark, or the default B-Scan image for the remainder of the work of this paper. The objective of the remainder of the work is to investigate whether an A-Scan matrix $A_{b r}^{*}$ can be achieved by performing some transformation to $A_{b r}$, so that $A_{b r}^{*}$ produces an enhanced B-Scan image having more contrast and/or detail, than the benchmark B-Scan image in Fig. 6b) which was produced by $A_{b r}$. Several experiments were performed on $A_{b r}$ to investigate this matter, and they are detailed in the remainder of this section. 


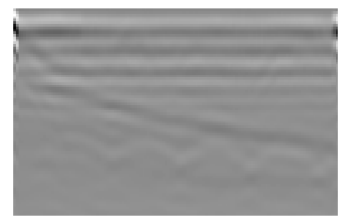

(a)

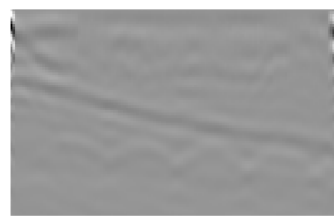

(b)
Fig. 6. Benchmark B-Scan image: (a) B-Scan from raw signal (generated from matrix $A$ ); (b) B-Scan from background subtracted signal (generated from matrix $A_{b r}$ ).

\section{B. Experiment 1: Absolute A-Scan}

The first experiment was taking the absolute value of the background removed A-Scan. This was done as follows by taking the absolute value of every element of $A_{b r}$ :

$$
A_{b r}^{*}=\left|A_{b r}\right|
$$

The resulting B-Scan is shown in Fig 7 b) along with the benchmark B-Scan in Fig. 7(a). It is evident when comparing Fig 7(b) with Fig 7(a), that the Absolute A-Scan transformation has not been successful in enhancing the benchmark B-Scan.

\section{Experiment 2: Exponential of $A-S c a n$}

In the second experiment the B-Scan image was generated after taking the exponential value of every element of the background removed A-Scan matrix. The operation is denoted as follows:

$$
A_{b r}^{*}=\exp \left(A_{b r}\right)
$$

The B-Scan that resulted from $A_{b r}^{*}$ generated in this manner is shown in Fig 7(c). It is evident when comparing Fig 7lc) with Fig 7(a), that the Exponential of A-Scan transformation too has not been successful in enhancing the benchmark B-Scan.

\section{Experiment 3: Exponential of Normalized A-Scan}

In the third experiment the B-Scan image was generated after taking the exponential value of every element of the normalized background removed A-Scan matrix. The operation is denoted as follows:

$$
A_{b r}^{*}=\exp \left[\frac{1}{\max \left(A_{b r}\right)} \times A_{b r}\right]
$$

It should be noted that the operation $\max \left(A_{b r}\right)$ in eq. 8 extracts the maximum value present in $A_{b r}$. The $\mathrm{B}-\mathrm{Scan}$ that resulted from $A_{b r}^{*}$ generated in this manner is shown in Fig 7(d). Unlike the results from Experiments 1 and 2, the Exponential of Normalized A-Scan transformation has produced a fair B-Scan. When comparing Fig 7(d) with Fig 7(a), one might observe that the B-Scan in Fig 7.d) has more clarity and contrast than Fig 7(a), with the slant Gypsum-Concrete interface and the parabolas considerably visible. Thus, one might argue that the Exponential of Normalized A-Scan transformation has produced a slightly enhanced B-Scan image.

\section{E. Experiment 4: Convolution of A-Scan with Normal Distri- bution PDF}

To generate $A_{b r}^{*}$ under Experiment 4, we first define the following: Suppose a row vector $C=\left[c_{1}, c_{2}, \ldots, c_{i}, \ldots c_{k}\right]$ exists such that $1 \leq i \leq k ; i, k \in \mathbb{Z}^{+}$; and $c_{i} \in \Re \forall i$, where $c_{1}=-n / 2$ and $c_{k}=n / 2$, where $n \in \mathbb{Z}^{+}$, and $c_{i+1}-c_{i}=1$ $\forall i$. Now suppose we specify a row vector $C$ as such, along with an arbitrary value each for $\mu\left(\in \Re_{0}^{+}\right)$and $\sigma\left(\in \Re^{+}\right)$of eq. 2. Hence, we define a row vector-shaped kernel $P(C)$ as follows:

$$
P(C)=\left[P\left(c_{1}\right), P\left(c_{2}\right), \ldots, P\left(c_{i}\right), \ldots, P\left(c_{k}\right)\right],
$$

where $P\left(c_{i}\right)$ is computed in a sense of probability mass using eq. $2 \forall i$. Thereon, we generate $A_{b r}^{*}$ as in eq. 10 following discrete $2 \mathrm{D}$ convolution as presented in eq. 3 and 4

$$
A_{b r}^{*}=A_{b r} * P(C)
$$

To generate $A_{b r}^{*}$ as in eq. 10 , the parameters $n\left(\in \mathbb{Z}^{+}\right), \mu$, and $\sigma$ need to be defined. For simplicity, we set $\mu=0$ and $\sigma=n$. Fig 7(e) shows the $\mathrm{B}-\mathrm{Scan}$ which results from $A_{b r}^{*}$ computed by setting $n=5$. When comparing with $\mathrm{B}-\mathrm{Scans}$ in Fig 7 (a) and Fig 7(d), one might argue that the Convolution of A-Scan with Normal Distribution PDF operation done in this experiment has achieved quite superior enhancement of the B-Scan.

Since the final B-Scan produced by this method depends on the value of $n$, a further experiment was done to evaluate the performance of this method by varying the value of $n$. Fig. 8 shows the obtained results for some $n$ values (i.e., $n=1,5,10$ and 100), along with the benchmark B-Scan for comparison. It can be seen from Fig. 8 that the results are quite positive for the low values of $n$ (e.g., $n=1$ and 5 while $n=5$ being the best) while the B-Scan loses quality as $n$ gets larger (e.g., $n=10$ and 100).

\section{F. Experiment 5: Convolution of A-Scan with Exponential of Normal Distribution PDF}

The manner in which $A_{b r}^{*}$ is generated through this method is quite similar to that of Experiment 4. The difference from Experiment 4 lies in the kernel for convolution in this method being the exponential of $P(C)$ (i.e., $\exp [P(C)]$ ) - meaning the exponential value of each element of $P(C)$ is computed and assigned to the kernel as opposed to the kernel being $P(C)$ in Experiment 4. The operation can be expressed as:

$$
A_{b r}^{*}=A_{b r} * \exp [P(C)]
$$

Fig 7 (f) shows the B-Scan which results from $A_{b r}^{*}$ computed by setting $n=5$. When comparing with B-Scans in Fig 7 (a) and Fig 7.d), one might argue that the Convolution of A-Scan with Exponential of Normal Distribution PDF method too has achieved quite superior enhancement of the B-Scan and appears quite identical in performance to the Convolution of A-Scan with Normal Distribution PDF method executed in Experiment 4.

Since the final B-Scan produced by this method too (like in Experiment 4) depends on the value of $n$, a further experiment 


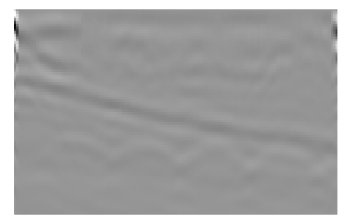

(a)

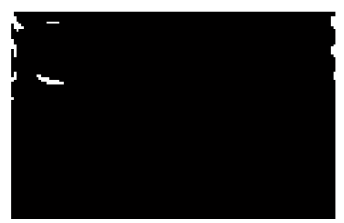

(c)

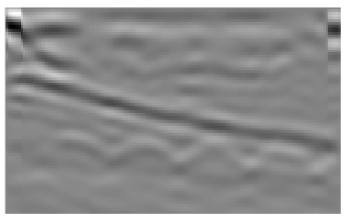

(e)

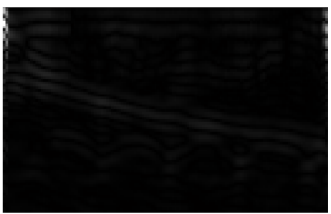

(b)

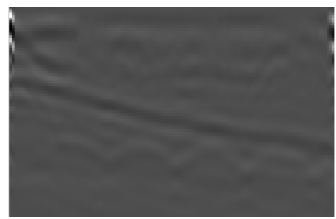

(d)

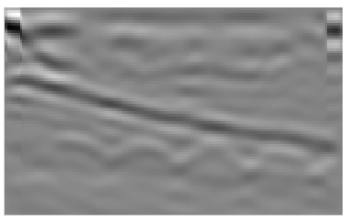

(f)

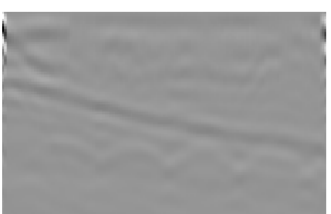

(a)

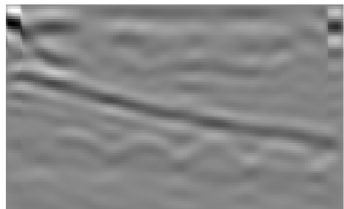

(c)

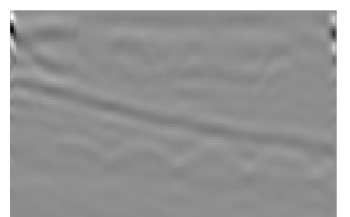

(b)

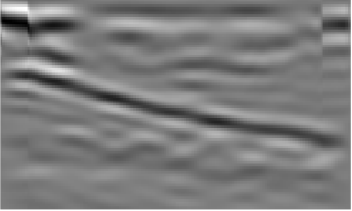

(d)

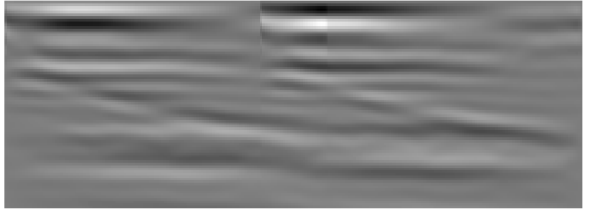

(e)

Fig. 7. Comparison of results: (a) Benchmark B-Scan; (b) B-Scan from Absolute A-Scan (Experiment 1); (c) B-Scan from Exponential of A-Scan (Experiment 2); (d) B-Scan from Exponential of Normalized A-Scan (Experiment 3); (e) B-Scan from Convolution of A-Scan with Normal Distribution PDF (Experiment 4); (f) B-Scan from Convolution of A-Scan with Exponential of Normal Distribution PDF (Experiment 5).

was done to evaluate the performance of this method by varying the value of $n$. Fig. 9 shows the obtained results for some $n$ values (i.e., $n=1,5,10$ and 100), along with the benchmark B-Scan for comparison. It can be seen from Fig. 9 that the results are quite positive for the low values of $n$ (e.g., $n=1$ and 5 while $n=5$ being the best) while the B-Scan loses quality as $n$ gets larger (e.g., $n=10$ and 100)-almost identical to what was observed with the results from Experiment 4.

\section{DisCuSSION AND CONCLUSIONS}

Enhancement of GPR B-Scan (grey-scale) images achievable through some convolution and scale transformations applied on A-Scans was investigated. The effect of enhancement was demonstrated against a benchmark B-Scan image created by mapping A-Scan wave amplitudes directly to grey-scale. Convolution-based methods were able to produce superior enhancement. The reason behind this could be the row vector-shaped kernels defined in this paper, being able to make use of adjacent A-Scan waves to good effect. Results suggest that through careful selection of such kernels and their parameter values, some enhancement in GPR images can be achieved even when the waves are affected by undesired factors such as moisture. Comparing the performance of such kernels against other techniques available for GPR image enhancement, and investigating on more kernels that may be effective on different applications, and prescribing protocols to tune their parameters could create interesting avenues for further research. Developing GPR-based robotic condition assessment tools, similar to those based on other
Fig. 8. Influence of $n$ on the results of Experiment 4: (a) Benchmark B-Scan; (b) B-Scan $(n=1)$; (c) B-Scan $(n=5)$; (d) B-Scan $(n=10)$; (e) B-Scan $(n=100)$.

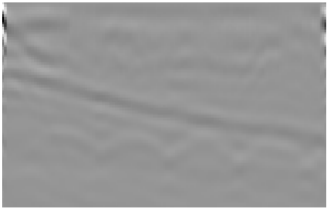

(a)

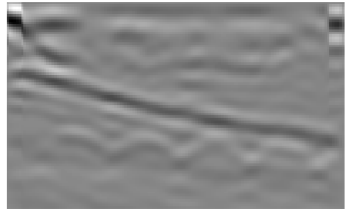

(c)

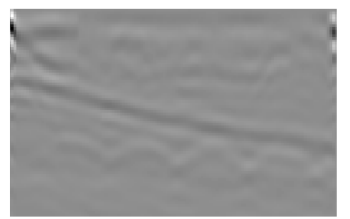

(b)

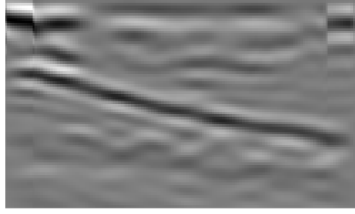

(d)

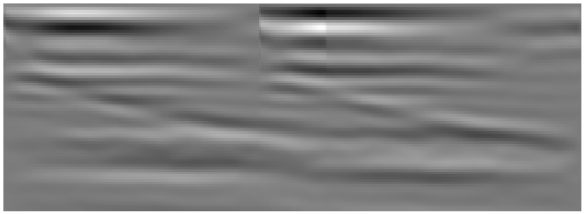

(e)

Fig. 9. Influence of $n$ on the results of Experiment 5: (a) Benchmark B-Scan; (b) B-Scan $(n=1)$; (c) B-Scan $(n=5)$; (d) B-Scan $(n=10)$; (e) B-Scan $(n=100)$.

sensing techniques done for water pipe assessment [30, 31], could also be an interesting avenue, especially for challenging applications such as sewer condition assessment.

\section{ACKNOWLEDGMENT}

This publication is an outcome from the 'Development of Sensor Suites and Robotic Deployment Strategies for Condition Assessment of Concrete Sewer Walls' project funded by the Sydney Water Corporation. 


\section{REFERENCES}

[1] L. B. Conyers, "Ground penetrating radar," Encyclopedia of Imaging Science and Technology, 2002.

[2] H. M. Jol, Ground penetrating radar theory and applications. elsevier, 2008.

[3] M. Beres Jr and F. Haeni, "Application of ground-penetrating-radar methods in hydrogeologie studies," Groundwater, vol. 29, no. 3, pp. 375-386, 1991.

[4] Z. Harari, "Ground-penetrating radar (gpr) for imaging stratigraphic features and groundwater in sand dunes," Journal of applied Geophysics, vol. 36, no. 1, pp. 43-52, 1996.

[5] C. S. Bristow and H. M. Jol, "An introduction to ground penetrating radar (gpr) in sediments," Geological Society, London, Special Publications, vol. 211, no. 1, pp. 1-7, 2003

[6] S. Laurens, J.-P. Balayssac, J. Rhazi, and G. Arliguie, "Influence of concrete relative humidity on the amplitude of ground-penetrating radar (gpr) signal," Materials and Structures, vol. 35, no. 4, pp. 198-203, 2002.

[7] C. Maierhofer, "Nondestructive evaluation of concrete infrastructure with ground penetrating radar," Journal of Materials in Civil Engineering, vol. 15, no. 3, pp. 287-297, 2003.

[8] J. Bungey, "Sub-surface radar testing of concrete: a review," Construction and Building materials, vol. 18, no. 1, pp. 1-8, 2004.

[9] K. Thiyagarajan, S. Kodagoda, R. Ranasinghe, D. Vitanage, and G. Iori, "Robust sensing suite for measuring temporal dynamics of surface temperature in sewers," Scientific Reports, vol. 8, no. 16020, 2018.

[10] K. Thiyagarajan, S. Kodagoda, L. Nguyen, and S. Wickramanayake, "Gaussian markov random fields for localizing reinforcing bars in concrete infrastructure," in 35th International Symposium on Automation and Robotics in Construction. IAARC, 2018, pp. 1052-1058.

[11] K. Thiyagarajan, S. Kodagoda, and J. K. Alvarez, "An instrumentation system for smart monitoring of surface temperature," in 2016 14th International Conference on Control, Automation, Robotics and Vision (ICARCV). IEEE, 2016, pp. 1-6.

[12] K. Thiyagarajan, S. Kodagoda, and L. Van Nguyen, "Predictive analytics for detecting sensor failure using autoregressive integrated moving average model," in 2017 12th IEEE Conference on Industrial Electronics and Applications (ICIEA). IEEE, 2017, pp. 1926-1931.

[13] K. Thiyagarajan, "Robust sensor technologies combined with smart predictive analytics for hostile sewer infrastructures," Ph.D. dissertation, 2018.

[14] K. Thiyagarajan, S. Kodagoda, L. Van Nguyen, and R. Ranasinghe, "Sensor failure detection and faulty data accommodation approach for instrumented wastewater infrastructures," IEEE Access, vol. 6, pp. $56562-56574,2018$.

[15] K. Thiyagarajan, S. Kodagoda, and N. Ulapane, "Data-driven machine learning approach for predicting volumetric moisture content of concrete using resistance sensor measurements," in 2016 IEEE 11th Conference on Industrial Electronics and Applications. IEEE, 2016, pp. 1288-1293.

[16] J. K. Alvarez, S. Sutjipto, and S. Kodagoda, "Validated ground penetrating radar simulation model for estimating rebar location in infrastructure monitoring," in Industrial Electronics and Applications (ICIEA), 2017 12th IEEE Conference on. IEEE, 2017, pp. 1460-1465.

[17] J. K. Alvarez and S. Kodagoda, "Application of deep learning imageto-image transformation networks to gpr radargrams for sub-surface imaging in infrastructure monitoring," in 2018 13th IEEE Conference on Industrial Electronics and Applications (ICIEA). IEEE, 2018, pp. 611-616.

[18] E. M. Johansson and J. E. Mast, "Three-dimensional ground-penetrating radar imaging using synthetic aperture time-domain focusing," in $\mathrm{Ad}$ vanced Microwave and Millimeter-Wave Detectors, vol. 2275. International Society for Optics and Photonics, 1994, pp. 205-215.

[19] E. Yigit, S. Demirci, C. Ozdemir, and A. Kavak, "A synthetic aperture radar-based focusing algorithm for b-scan ground penetrating radar imagery," Microwave and Optical Technology Letters, vol. 49, no. 10, pp. 2534-2540, 2007.

[20] R. Stolt, "Migration by fourier transform," Geophysics, vol. 43, no. 1, pp. 23-48, 1978.

[21] E. Fisher, G. A. McMechan, A. P. Annan, and S. W. Cosway, "Examples of reverse-time migration of single-channel, ground-penetrating radar profiles," Geophysics, vol. 57, no. 4, pp. 577-586, 1992.

[22] C. J. Leuschen and R. G. Plumb, "A matched-filter-based reversetime migration algorithm for ground-penetrating radar data," IEEE
Transactions on Geoscience and Remote Sensing, vol. 39, no. 5, pp. 929-936, 2001.

[23] S. C. Fisher, R. R. Stewart, and H. M. Jol, "Ground penetrating radar (gpr) data enhancement using seismic techniques," Journal of Environmental and Engineering Geophysics, vol. 1, no. 2, pp. 89-96, 1996.

[24] C.-S. Chen and Y. Jeng, "Nonlinear data processing method for the signal enhancement of gpr data," Journal of Applied Geophysics, vol. 75 , no. 1, pp. 113-123, 2011.

[25] L. Liu, K. He, X. Xie, and J. Du, "Image enhancement with waveequation redatuming: application to gpr data collected at public transportation sites," Journal of Geophysics and Engineering, vol. 4, no. 2, p. 139, 2007.

[26] M. E. Yavuz, A. E. Fouda, and F. L. Teixeira, "Gpr signal enhancement using sliding-window space-frequency matrices," Progress In Electromagnetics Research, vol. 145, pp. 1-10, 2014.

[27] L. E. Besaw and P. J. Stimac, "Deep learning algorithms for detecting explosive hazards in ground penetrating radar data," in Detection and Sensing of Mines, Explosive Objects, and Obscured Targets XIX, vol. 9072. International Society for Optics and Photonics, 2014, p. 90720Y.

[28] A. Kalogeropoulos, J. Van der Kruk, J. Hugenschmidt, S. Busch, and K. Merz, "Chlorides and moisture assessment in concrete by gpr full waveform inversion," Near Surface Geophysics, vol. 9, no. 3, pp. 277285,2011

[29] S. Laurens, J. Balayssac, J. Rhazi, G. Klysz, and G. Arliguie, "Nondestructive evaluation of concrete moisture by gpr: experimental study and direct modeling," Materials and structures, vol. 38, no. 9, pp. 827832, 2005.

[30] J. V. Miro, D. Hunt, N. Ulapane, and M. Behrens, "Towards automatic robotic ndt dense mapping for pipeline integrity inspection," in Field and Service Robotics. Springer, 2018, pp. 319-333.

[31] J. Valls Miro, N. Ulapane, L. Shi, D. Hunt, and M. Behrens, "Robotic pipeline wall thickness evaluation for dense nondestructive testing inspection," Journal of Field Robotics, vol. 35, no. 8, pp. 1293-1310, 2018. 